\title{
DU PROTECTORAT À L'ANNEXION
}

La lente «pacification » des Îles Sous-le-Vent (Polynésie), 1880-1897

Renaud Meltz

Armand Colin | Monde(s)»

$2013 / 2 \mathrm{~N}^{\circ} 4 \mid$ pages 233 à 250

ISSN 2261-6268

ISBN 9782200928629

Article disponible en ligne à l'adresse :

https://www.cairn.info/revue-mondes1-2013-2-page-233.htm

Distribution électronique Cairn.info pour Armand Colin.

(C) Armand Colin. Tous droits réservés pour tous pays.

La reproduction ou représentation de cet article, notamment par photocopie, n'est autorisée que dans les limites des conditions générales d'utilisation du site ou, le cas échéant, des conditions générales de la licence souscrite par votre établissement. Toute autre reproduction ou représentation, en tout ou partie, sous quelque forme et de quelque manière que ce soit, est interdite sauf accord préalable et écrit de l'éditeur, en dehors des cas prévus par la législation en vigueur en France. Il est précisé que son stockage dans une base de données est également interdit. 


\section{Du protectorat à l'annexion}

\section{La lente « pacification » des Îles Sous-le-Vent (Polynésie), 1880-1897}

\section{Renaud Meltz}

Université de la Polynésie française

\section{Résumé}

La Convention de Jarnac, qui réglait depuis 1847 la rivalité franco-britannique dans les Îles Sous-le-Vent, en interdisant aux deux puissances de se saisir de cette région, a été abrogée en 1887, permettant à la France de proclamer l'annexion de cet archipel polynésien, en 1888. L'arrivée de plusieurs centaines d'hommes, en 1897, permit de « pacifier » ce territoire, dont une partie des habitants refusait l'annexion. L'intervention militaire, asymétrique, fut le seul moyen de réduire la résistance à la colonisation, mais son souvenir continue à nourrir l'identité politique des Polynésiens.

Mots-clés : Polynésie française - Îles Sous-le-Vent Colonisation - Raiatea - Teraupoo.

\begin{abstract}
From Protectorate To Annexation, 1880-1897. The Slow "Pacification" of the Leeward Islands (French Polynesia)
\end{abstract}

The Jarnac Convention (1847) prohibiting France and Britain to seize the Leeward Islands was abrogated in 1887, allowing France to annex them in March 1888. Hundreds of French soldiers arrived in early 1897 to "pacify" this territory. The military intervention was the only mean to annihilate resistance to the French colonization, and memories of this resistance keep feeding the political identity of the Polynesians.

Keywords: French Polynesia-îles Sous-le-VentColonization - Raiatea - Teraupoo.

monde(s), $n^{\circ}$ 4, septembre 2013, p. 233-251 
$\mathrm{A}^{\mathrm{v}}$ vant l'annexion des Îles Sous-le-Vent (ISLV), les Établissements français de l'Océanie (EFO), soit l'ensemble formé par Tahiti, les Marquises et les Gambier, réunissaient quelque 25000 habitants au milieu des années 1880. Alors que les administrateurs coloniaux déploraient la dépopulation de ces territoires, les ISLV ne devaient ajouter à cet ensemble que quelques centaines d'habitants par île. On les estime à 1500 pour Raiatea, la plus grande d'entre elles ${ }^{1}$. L'ensemble de ces îles, avec Tahaa, Bora Bora, Huhaine et Maupiti, forme une surface de quelque $450 \mathrm{~km}^{2}$.

Autant dire que les ISLV ne représentaient pas un enjeu par leur taille ou leurs ressources démographiques, par les perspectives d'exploitation agricole ou de peuplement qu'elles laissaient espérer. Isolé, cet ensemble appartenait pourtant à plusieurs ensembles régionaux, ce qui lui conférait un intérêt stratégique. Traditionnellement, il s'inscrivait dans un système de relations internationales qui l'intégrait à l'ensemble polynésien, soit le triangle formé au nord par Hawaï, à l'ouest par la NouvelleZélande et à l'est par l'île de Pâques : ses marae en constituaient le centre religieux ${ }^{2}$.

1 Pas plus de 2000 , en tout cas, puisqu'en 1897, les opérations militaires terminées, chaque habitant reçut " un papier servant de passeport et portant son nom, et un numéro allant de 1 à 1999 ", Service historique de la Défense (SHD), BB4 1603. Les différents dossiers consultés, très riches, ne sont pas foliotés mais classés par ordre chronologique.

2 Les marae sont des temples en plein air, qui structurent la vie religieuse, sociale et politique. Le marae de
Les administrateurs français avaient conscience de cette situation exceptionnelle. On trouve dans la documentation du chef de la station du Pacifique un rapport non daté, très bien informé sur la question, rédigé par le lieutenant de vaisseau en retraite Xavier Caillet (1822-1901). Cet ancien inspecteur des affaires indigènes, qui avait inspiré la première demande de protectorat des chefs des ISLV à la France, en 1880, éclairait les marins sur le prestige particulier de l'île :

« La suprématie de Raiatea sur toutes les autres îles de l'archipel est traditionnelle, ainsi que la supériorité de ses guerriers : c'est ce qui explique l'étendue de cette île sur la carte faite par le commandant espagnol Bonechea d'après les données fournies par l'imagination tahitienne de 1772. C'est dans un district de cette île sainte à Opoa, que se trouve le plus ancien Marae de toute cette partie de la Polynésie $»^{3}$.

Des relations familiales et des alliances étroites liaient les ISLV à d'autres îles, notamment à Tahiti : c'est à Raiatea que la reine tahitienne Pomare IV s'était réfugiée, lorsqu'elle avait contesté la souveraineté française pendant la guerre franco-tahitienne des années 18441846 après s'être résolue, sous la contrainte, à

Taputapuatea possède un prestige international, dans le monde polynésien. Voir notamment Teuira Henry, Tahiti aux temps anciens, Paris, Société des océanistes, 2004 ; Philippe Bachimon, Tahiti entre mythes et réalité. Essai d'histoire géographique, Paris, Éditions du CTHS, 1990.

3 SHD, BB4 1602. Xavier Caillet était désormais conseiller général à Tahiti. 
solliciter la protection de la France, en $1842^{4}$. Si la Convention dite de Jarnac, qui régla la rivalité franco-anglaise en 1847, a longtemps séparé les deux îles, en interdisant de les associer dans la même souveraineté, les liens demeurèrent étroits.

Raiatea appartenait aussi, à bien des égards, à cette Océanie britannique qui glissait dans l'Empire informel sous l'influence du protestantisme anglais. Les étrangers qui résidaient à Raiatea lors de sa prise de possession par la France, en 1888, étaient très minoritairement français, si l'on s'en tient aux listes établies par les marins, qui comptaient une bonne trentaine de foyers anglo-saxons pour seulement quatre Français.

Lî̂le, enfin, appartenait à une économie régionale difficile à se représenter, qui l'intégrait toutefois à une aire Pacifique assez vaste pour que la seule monnaie qui y eût cours fût le piastre chilien.

Pour autant, les ISLV possédaient leur unité propre. Elles disposaient de leur gouvernement, plus ou moins commun à Raiatea et Tahaa, qui partageaient le même lagon, avec son système de monarchie souple inspiré par le modèle anglais que les missionnaires de la London Mission Society avaient diffusé, mais dont la pratique demeurait proche du système traditionnel, où le « roi » était choisi par les chefs.

4 Je me permets de renvoyer à deux de mes articles sur la question : Renaud Meltz, « Le protectorat français sur Tahiti », Revue d'histoire diplomatique, 2011/2 ; « L'affaire Pritchard ", Revue d'histoire diplomatique, 2012/1.
Une dynastie, celle des Tamatoa, se maintenait, en dépit de quelques accidents individuels. Tamatoa V, fils cadet de la reine Pomare, avait été adopté par Tamatoa IV, son grand-oncle ${ }^{5}$. En 1884, Tamatoa VI, petit-fils de Tamatoa IV avait été élu. Il lui revint d'affronter la période tourmentée de la « pacification » des ISLV, face à des Français qui ne l'appréciaient guère : «Très soumis à l'influence des missionnaires anglais, on le dit peu disposé pour nous », observait un marin dès le début de son règne. Après son abdication, Tuarii, de la même lignée (c'était la fille du roi Tahitoe régnant en 1880 ), devint reine de ce que nous appellerons le « tiers parti », qui se tenait entre les partisans des Français et les rebelles.

Que faut-il entendre par la « pacification » des ISLV?

On désigne par là les dix années séparant la proclamation de l'annexion des ISLV, en mars 1888, une fois levée l'hypothèque anglaise, sur le plan de la diplomatie européenne, de l'annexion effective, au début de l'année 1897, lorsqu'une opération mobilisa plusieurs centaines d'hommes, débarqués sur l'île pour réduire les quelques centaines de rebelles à la souveraineté française.

Le mot de «pacification », employé parfois par les acteurs occidentaux (on ne sait

5 Sur cette dynastie, voir Bruno Saura, Huahine aux temps anciens, Service de la culture et du patrimoine de Polynésie française, 2005 ; Bruno Saura, La lignée royale des Tamatoa de Ra'iatea (Îles Sous-le-Vent), Papeete, ministère de la Culture, 2003. 
pas comment en parlaient les autochtones), recouvre plusieurs notions.

La dimension militaire est évidente ; il s'agit d'une guerre asymétrique, marquée par la supériorité technique des Français, armés de fusils et soutenus par l'artillerie de marine. Par le nombre, la connaissance du terrain, qualifié de " brousse », et l'adaptation au climat, les soldats français se trouvaient en revanche en situation d'infériorité. Avant d'aborder Raiatea, l'aspirant Henri de Menthon exprime cette appréhension :

« Dans la brousse nos soldats n'ont pas l'avantage, ils redoutent toujours une embuscade, l'attaque d'ennemis invisibles, les balles qui tuent par surprise, tous les imprévus de la guerre de halliers qui rendent timides les plus braves $»^{6}$.

Cette guerre qui ne dit pas son nom revient officiellement à une opération de police, dans un espace désorganisé par la rivalité internationale et les dissensions internes. La présence de planteurs, artisans et commerçants étrangers, leurs activités, leurs revenus, justifiant des taxes portuaires et des patentes, bref, l'intégration de l'île dans un espace commercial plus vaste, avaient suscité de nouvelles occasions de rivalités entre les chefs et les districts.

Ces déséquilibres, provoqués par un contact tardif et violent, du fait du choc microbien notamment, entre des civilisations qui s'ignoraient jusqu'à la fin du XVIII ${ }^{\mathrm{e}}$ siècle, confèrent aussi un

6 Henri de Menthon, Vingt-deux mois de campagne autour du monde. Journal d'un aspirant de marine, Paris, Plon, 1900. sens culturel à la pacification des ISLV : imposition d'un ordre civil, administratif, et d'une vision du monde ${ }^{7}$.

Deux visions du monde, deux cultures matérielles, deux systèmes d'exploitation du monde naturel se sont rencontrés, mais cette dualité n'a pas toujours été contradictoire et a pu entraîner des collaborations aussi bien que des conflits. Les autochtones avaient besoin des armes à feu occidentales pour tenir tête aux Français, comme une faction, à Tahiti, en avait profité pour unifier l'île à son bénéfice (la dynastie des Pomare). La supériorité des embarcations occidentales, plus grandes, plus rapides, n'a pas empêché que les Français dépendent de la culture maritime des autochtones : les grands bâtiments avaient besoin d'être guidés par les pilotes locaux à travers les passes piégeuses qui conduisent de l'Océan aux lagons. Les quiproquos culturels n'ont pas empêché l'élaboration de langages communs ; ce fut le cas, par exemple, de la symbolique des pavillons nationaux.

Reste que le mot de « pacification » est tellement en décalage avec les dix années de blocus et l'opération militaire finale, que l'expression n'a pas fait florès pour la postérité : on parle couramment, aux îles, de " guerre des ISLV ».

Compte tenu de l'étroitesse de l'historiographie sur cette question, ma contribution se veut

7 Les Français maintiennent, certes, le code local, mais il est lui-même marqué par les missionnaires protestants et s'inspire de la Bible au point qu'on parle parfois, pour certaines îles, de « théocraties ". 
essentiellement, au premier niveau d'analyse, un effort de rationalisation des événements et de mise au clair de la chronologie ${ }^{8}$. Le rythme lent du processus de " pacification », avant la conquête effective, tient sans doute à des raisons générales et oblige à se tenir à une échelle assez large. Celle des relations internationales, la politique de la France en Océanie s'inscrivant dans un vaste marché des acquisitions coloniales ; celle des moyens militaires, qui commandait la politique impériale. La question des ISLV relève davantage de la haute politique internationale que d'enjeux de politique intérieure, en France, où l'on ne discute guère de Raiatea. Même à Tahiti, on semble considérer la situation des ISLV avec un mélange d'indifférence, d'ironie et d'irritation.

Quant à la situation sur le terrain, la très grande asymétrie des traces n'aide pas à adopter le point de vue des autochtones, mais l'échelle locale donne au moins à voir comment les pacificateurs, marins et civils, se les représentaient.

\section{La rivalité franco-anglaise}

Depuis la Convention de Jarnac, en 1847, jusqu'à la proclamation du protectorat sur les ISLV en 1880 , et l'abrogation de la Convention de Jarnac, en 1887, trente années furent consacrées à une

8 Il existe néanmoins une thèse fort précieuse sur Raiatea : Anne-Lise Pasturel, « Raiatea 1818-1945 : permanences et ruptures politiques, économiques et culturelles ", thèse de doctorat en histoire sous la direction de Jean-Marc Regnault, Université de la Polynésie française, 2000, 3 vol., $517 \mathrm{p}$. colonisation larvée, préparant les dix années de pacification proprement dite, de 1887 à 1897.

Dans un premier temps, il s'agissait moins d'intégrer les ISLV à une Polynésie française encore inexistante, au-delà de l'ensemble TahitiMoorea et de l'archipel des Marquises, que d'empêcher l'arrivée d'une nouvelle puissance impériale comme l'Allemagne. Plus tard, la III ${ }^{\mathrm{e}}$ République trouva de nouvelles raisons d'intégrer les ISLV à ses possessions océaniennes : la cohérence stratégique, le percement du canal de Panama et le rôle d'escale sur la route de l'Australie, tout cela nourrissait un nouvel élan colonial, dans le contexte de la colonisation de Madagascar et du Tonkin.

L'initiative fut d'abord locale, suivant une logique fonctionnelle de la part des représentants français qui voulaient étendre leur autorité à l'ensemble d'une région ayant sa cohérence. Elle répondait également à la pression des intérêts étrangers : commerciaux, voire coloniaux de la part d'une Allemagne déjà tentée par les premières velléités d'une Weltpolitik; stratégiques, pour l'Australie et la Nouvelle-Zélande, où l'on considérait les archipels océaniens comme des dépendances naturelles; voire une question de prestige pour une puissance issue d'un empire colonial comme le Chili, qui s'emparait de l'île de Pâques, dédaignée par la France. La Grande-Bretagne, dont l'impérialisme demeurait vivace dans la région, et dont les missionnaires s'inquiétaient 
continûment de l'arrivée d'une concurrence catholique, continuait de veiller au grain.

Une logique de clientèle, nourrie par la division en clans et les alliances passées entre les notables tahitiens et les chefs des ISLV, instaura naturellement la France en arbitre de la vie locale, en dépit de la Convention de Jarnac ${ }^{9}$. La mort de Pomare IV, en 1877 - occasion du passage du protectorat à l'annexion pure et simple de Tahiti, en 1880 -, modifia la donne, alors que la présence allemande se faisait plus menaçante aux ISLV. Le ministère de la Marine et des Colonies envoya Isidore Chessé, chef de bureau du ministère, en qualité de Commissaire civil du protectorat, pour établir l'annexion de Tahiti et régler le cas des ISLV, ayant représenté au Quai d'Orsay que la Convention de Jarnac ne prévoyait pas le cas d'une prise de possession par une puissance tierce. Chessé reçut le soutien des pasteurs de Tahiti et, partant, de celui de Raiatea. Les chefs des ISLV, inquiets que la présence commerciale allemande devienne ingérence politique, acceptèrent la protection de la France, sans voir, peut-être, qu'ils s'engageaient dans un processus d'annexion. $\mathrm{Au}$ printemps 1880, Tahitoe, roi de Raiatea et de Tahaa, sollicita la protection de la France pour les affaires extérieures dans un document cosigné par une douzaine de chefs et orateurs ; quelques mois plus tard, le même se plaignit aux Britanniques qu'on lui avait extorqué ce

9 Jean-François Baré, Tahiti, les temps et les pouvoirs. Pour une anthropologie historique du Tahiti post-européen, Paris, Éditions de l'Orstom, 1987, p. 317. document sous la menace d'une intervention militaire allemande ${ }^{10}$. Le pavillon du protectorat provisoire avait toutefois été adopté.

À Paris, le protectorat ne fut pas mieux accueilli, d'abord: Chessé fut désavoué par Freycinet, ministre des Affaires étrangères, qui attendait un accord général avec la Grande-Bretagne et l'Allemagne pour trancher la question. L'application du protectorat était suspendue à l'abrogation de la Convention de Jarnac. Une conférence s'ouvrit à Londres, en mai 1881 ; elle n'aboutit pas au règlement général escompté, mais la Grande-Bretagne consentit à un protectorat provisoire de six mois, renouvelable. C'est le 16 novembre 1887, seulement, que la Grande-Bretagne accepta le principe de l'abrogation de la Convention de Jarnac, en échange d'une évacuation des forces armées françaises aux Nouvelles-Hébrides (Vanuatu). L'abrogation de la Convention fut conclue le 30 mai 1888, au grand dam de l'Australie et de la Nouvelle-Zélande, qui craignaient moins l'abandon des ISLV que celui des Nouvelles-Hébrides. Cet accord complétait celui que la France avait passé avec l'Allemagne, le 24 décembre 1885, pour partager des zones d'influence en Afrique, contre renonciation des ambitions allemandes en Océanie, dans les zones convoitées par les Français ${ }^{11}$.

10 lbid., p. 318.

11 Anne-Lise Pasturel, « Raiatea 1818-1945 », op. cit., p. 313 (cf. note 8). 


\section{La révolte d'une partie des autorités locales}

Le protectorat de 1880 se trouvait régularisé sur le plan du droit international et préparait l'annexion, mais une partie des chefs contesta l'immixtion française, dès le deuxième semestre 1887.

La France fut d'abord impliquée comme arbitre d'une querelle opposant deux de ses clients, sur fond de rivalité entre Tahaa et Raiatea. D'un côté, Tavana, le régent ou vice-roi de Tahaa, favorable aux Français, décoré de la Légion d'honneur en mai $1890^{12}$. De l'autre, Peu, orateur du roi de Raiatea, mais surtout pilote - « l'homme le plus intelligent de ces îles » au dire des marins ${ }^{13}$.

Alors que la justice locale avait condamné Peu et l'avait déchu de son rôle de pilote, les Français le rétablirent dans ses fonctions et lui accordèrent de surcroît la nationalité française ${ }^{14}$. Dans ce contexte, cinq chefs de Raiatea signèrent une pétition, le 25 septembre 1887, réclamant l'installation d'un résident français comme garant de l'ordre public. Ces chefs furent aussitôt pris à partie et exilés par ceux qui, au contraire, contestaient le protectorat français : Meahao, mais surtout Teraupoo, autour de qui devait se cristalliser le refus de l'impérialisme français ${ }^{15}$.

12 SHD, BB4 1604.

13 Ibid., 1602.

14 SHD, BB4 1585, journal du commandant supérieur aux ISLV.

15 Le révérend Richards raconte les faits au consul britannique dans une lettre du 30 septembre 1887, archives de la mission de Londres, citées par Anne-Lise Pasturel, « Raiatea 1818-1945 », op. cit., p. 316 (cf. note 8).
Qui était Teraupoo ? L'historien est confronté à un flou qui participe de sa légende ; plutôt qu'un biographe, il a d'ailleurs trouvé un romancier pour écrire sa vie ${ }^{16}$. Si l'on est bien en peine d'en fournir une représentation fiable, au-delà de quelques clichés photographiques qui ne révèlent que des traits, des mensurations, et même une mise ordinaire dans ses vêtements occidentaux, c'est que les autorités civiles et militaires françaises ne s'étaient guère intéressées à sa trajectoire biographique, préférant en dresser un portrait psychologique généralement péjoratif, et conclure à l'impossibilité de traiter avec lui. Le capitaine de vaisseau Laguerre, le voyait « ardent, ambitieux, brouillon ${ }^{17}$.

Ce n'est que cinq ans après la proclamation de l'annexion qu'est produit le premier rapport - et dernier que nous ayons trouvé, portant sur ses motivations: "Noble patriotisme » ou «Ambition personnelle ${ }^{18}$ ? Le marin qui rédige ce rapport est aussi le premier à tenter une analyse de la stratégie du chef rebelle. Réflexion tout analogique, rapprochant sa méthode de celle des Anglais: les uns comme les autres tâteraient le terrain, allant aussi loin que possible, mais avec prudence - rapprochement qui tient à la fois d'une grille de lecture classique pour un marin français, éternel adversaire des Anglais, et de la proximité plus ou moins fantasmée des

16 Jean Dorsenne, Un fils de cannibales, Paris, Éditions de La Nouvelle Revue Critique, 1927 (livre achevé en 1924).

17 SHD, BB4 1600.

18 Ibid., 1604, juillet 1892. 
rebelles avec les pasteurs et les habitants anglo-saxons. En tout cas, une sorte de respect se dégage du portrait du chef rebelle, qui n'a pas les attributs prêtés en général aux «Canaques » comme les Occidentaux appellent les autochtones océaniens, mollesse, indolence, manque d'esprit de suite :

« En somme, Teraupo est un homme ambitieux et audacieux, persévérant, intelligent et prévoyant, qui, s'étant imposé par son énergie comme chef à la majeure partie de la population de Raiatea, se gardera bien de compromettre par des mesures imprudentes la haute situation qu'il a acquise $»^{19}$.

Pour autant, les marins observèrent, en cette même année 1892, que Teraupoo échoua à se faire proclamer souverain des habitants refusant la souveraineté française.

S'ensuivit un long épisode où les Français réclamèrent en vain la destitution des chefs rebelles par le roi, qui gagna du temps. Il n'en pouvait mais, ni ne souhaitait, sans doute, une hégémonie française. Confrontés à la partition de l'autorité, les marins évoquèrent d'emblée la menace de $«$ guerre civile $»^{20}$.

Dans ce début de crise, décisif, le contact entre les marins français et les autochtones se caractérise par des malentendus culturels, nourris par l'incompréhension linguistique.

19 ld.

20 Lettre du commandant du Scorpion à celui de l'Aorai, le 3 octobre 1887.
« Faute d'interprète, j'ai la plus grande difficulté à me faire comprendre du roi et des chefs, et il est plus que probable que ce qu'on me dit est aussi mal traduit que ce que je veux leur dire, de là des confusions et des contradictions continuelles »,

observe le commandant du Fabert. Ce qui n'empêche pas une lecture très réaliste des rapports de force et des objectifs réciproques. Les marins français maudissent "l'hypocrisie » des " braves canaques », qui dès " qu'ils verront le Fabert, c'est-à-dire la force, s'éloigner, deviendront ce qu'ils étaient : des fomenteurs de désordre et d'anarchie ». Ils les blâment de croire que la France a des visées annexionnistes, ce qui était naturellement son intention finale, par-delà les intermédiaires juridiques ${ }^{21}$.

Si les marins français se sont abusés, ce sont sur les pouvoirs réels du roi ("il n’a du Roi que le nom », a constaté un marin dépité), qu'ils se représentaient sur le modèle de la monarchie européenne. Le roi voulait bien laisser aux Français le soin d'arrêter Teraupoo, mais ne souhaitait pas les en saisir par écrit, attitude louvoyante qui exaspérait les partisans français. Un chef dit au roi : «Vous n'ignorez pas que lorsqu'un gouvernement a besoin du secours d'un autre gouvernement il le demande toujours par un écrit, et c'est ainsi qu'on agit dans tous les pays civilisés ». À ces mots, Tamatoa annonça son abdication au profit $\mathrm{du}$ vice-roi de Tahaa, condamnant implicitement

21 SHD, BB4 1602, octobre 1887, rapports du commandant du Fabert. 
la domination française et ses codes culturels : " Jusqu'à présent tous les écrits que nous avons adressés au Gouvernement français ou passés entre lui et mon Gouvernement, n'ont amené que des troubles et des malheurs dans notre pays ». Le roi ajouta qu'il avait attendu de la France qu'elle rétablisse l'ordre, ce qui n'était pas le cas. De fait, les Français se contentaient de raids navals, pour détruire les villages rebelles avec leur artillerie. La mise en place d'une garnison française à Raiatea, décidée à la mi-octobre, entérina une stratégie défensive pour protéger le pavillon du protectorat français $^{22}$.

En somme, au début de l'année 1888, alors que l'annexion n'était pas encore prononcée, le protectorat français, admis par la Grande-Bretagne, était déjà largement contesté sur place.

\section{Une annexion contestée}

\section{Annexion virtuelle et tentatives d'assimilation culturelle}

Suite à l'accord des Puissances, la prise de possession de jure des ISLV prit la forme d'une tournée du gouverneur des EFO, qui souhaitait procéder à des déclarations solennelles dans les différentes îles de l'archipel. Mais la tournée, en mars 1888, n'eut rien de triomphale. Le 17 mars, le gouverneur se rendit à Raiatea, et mit en place toute une liturgie politique, dont la

22 Ibid., le capitaine de vaisseau Laguerre, le 26 décembre 1888, au gouverneur des EFO. symbolique devait marquer les esprits. À suivre le récit de l'instituteur, peu de monde assista à la cérémonie. Rien ne résume mieux l'écart entre la proclamation virtuelle de l'annexion et la nécessité locale de " pacifier par les armes », que le symbole des cartouches improvisées avec les pages du Journal Officiel où l'annexion avait été annoncée ${ }^{23}$.

De décevante, à Raiatea, la tournée devint tragique, à Huahine : les marins furent accueillis par un feu nourri, qui fit trois morts chez les Français, dont un enseigne de vaisseau. Si les récits des marins, de l'instituteur et des pasteurs, offrent autant de versions différentes de l'incident, il en est une qui illustre remarquablement la dimension culturelle de la pacification : l'origine de l'échange de coups de feu proviendrait d'une baignade impudique d'un marin français. Autrement dit, c'est en transgressant l'ordre puritain venu de l'Occident, que l'Occidental avait mis le feu aux poudres :

«Le Commandant d'une goélette de guerre s'étant baigné sans caleçon, les indigènes l'injurient. Il ne veut pas être jugé suivant les lois indigènes, au contraire il demande à punir les hommes qui lui ont manqué de respect et les demande à son bord $»^{24}$.

Faute de réduire la résistance par la force, les autorités françaises usèrent de méthodes d'in-

23 «Édouard Jaulmes, premier instituteur à Raiatea. Relation du voyage et du séjour ", Journal de la société des océanistes, vol. 26, 1970, le 27 mars, p. 71.

24 Ibid., récit de Viénot à Jaulmes. 
tégration plus douces, en utilisant deux agents traditionnels de la colonisation : l'instituteur et l'homme d'église.

L'installation d'un premier instituteur français de 27 ans, Édouard Jaulmes, avait été prévue pour la fin de l'année 1887. Le principe en avait été décidé un an plus tôt, en vue de « répandre dans cet archipel l'influence française ainsi que la connaissance de notre langue $»^{25}$. La révolte différa son installation et le maintint pour quelques mois à Papeete. Débarqué finalement en mars 1888, trois semaines avant la proclamation de l'annexion, il fut bien accueilli par le pasteur anglais et put ouvrir son école.

Les Français ont trouvé en la personne du pasteur Richards un collaborateur de bonne volonté pour leur effort de pacification. Le 26 mars, l'instituteur nota dans son journal : « Les rebelles doivent attaquer si on n'enlève pas le pavillon français. M. Richards se décide malgré sa maladie d'aller voir lui-même Teraupoo le lendemain ». Les militaires, longtemps méfiants, se réjouirent de cette entremise. Ils félicitèrent le « ministre protestant anglais de s'être loyalement employé pour conseiller la soumission à un homme hostile aux Français ». Ils enregistraient son aide, bénéficiant des renseignements qu'il apportait; le 10 juin 1888, par exemple, lorsqu'il se rendit dans le district des rebelles :

« Le pasteur Richards appelé pour baptiser des enfants par les rebelles est allé [dans le district

25 SHD, BB4 1602. rebelle d'] Avera avec son cotre à 6 heures du matin et est revenu à 5 heures du soir. On n'a voulu répondre à aucune de ses questions [...]. La femme de Teraupoo dit qu'ils se défendront jusqu'à la mort. Ces gens sont fanatisés et considèrent Teraupoo comme invulnérable. Ils sont près du bord de la mer 300 ou 400 avec 4 grands pavillons de Raiatea ».

La question de la loyauté du protestantisme anglais à l'autorité française ne se posa plus, après la mort du pasteur Richards, puisqu'elle précipita le passage de témoin entre la London Mission Society anglaise et la Société des missions évangéliques de Paris (SMEP) française ${ }^{26}$.

Quant aux missionnaires catholiques, ils prétendirent au premier rôle dans leur collaboration à la pacification, soucieux de plaire aux autorités pour supplanter les protestants et valider, sous la III $^{\mathrm{e}}$ République, le sens d'une colonisation que Guizot avait justifiée, en son temps, par la vocation d'une puissance catholique à faire rayonner sa foi ${ }^{27}$.

\section{Partition de l'île et stratégie défensive}

Pendant la décennie qui suivit, la longue rébellion de Teraupoo réduisit la réalité de la souveraineté française à une partie de Raiatea, généralement divisée en deux ou trois ensembles. Uturoa, le principal port et lieu de résidence des Européens, fut doté d'un fort, construit par les

26 Journal de la SMEP, janvier 1889.

27 Archives de l'évêché de Papeete, lettre du Père Collette, au provincial picpusien, le 12 août 1897. 
marins, où mouillait le navire amiral de la flotte des ISLV. Quelques cases et un temple formaient le village, traversé par une route unique. Dans le district d'Avera, où s'exerçait l'autorité de Teraupoo, 400 à 600 hommes vivaient en bord de mer, la plupart dans cinq grandes cases communes, mais ils s'enfonçaient dans une gorge à l'accès difficile en cas de menace. Dans le district de Tevaitoa, une centaine d'hommes refusait également l'autorité française. Ces dissidents voulaient l'indépendance, voire la protection de la Grande-Bretagne. Entre les deux, dans une proportion flottante dans le temps, une bonne partie de la population vivait sous le régime intermédiaire et mouvant du protectorat. Les Français toléraient qu'ils arborent le drapeau dudit protectorat à côté des couleurs françaises, comme c'était le cas dans l'île sœur de Tahaa.

Pendant ces dix années de pacification virtuelle, la stratégie défensive se réduisit à la protection du pavillon français à Uturoa, comme symbole de la souveraineté, et au blocus maritime, qui reposait sur une flotte modeste : le Papeete et ses 25 hommes d'équipage, relevé par un navire équivalent, l'Aube qui, avec ses 129 hommes, venait ponctuellement, en cas de tension particulière. La séparation du ministère des Colonies de celui de la Marine suscita une grande prudence chez les marins. Le gouverneur avait autorité sur l'amiral mais, en cas d'incident sur place, le dernier mot revenait aux marins ${ }^{28}$. L'amiral, commandant la division

28 Plusieurs notes l'établissent, par exemple en SHD, BB4 1602, le 16 novembre 1885. navale de l'océan Pacifique, se voit régulièrement rappeler que « toute action militaire offensive ne peut plus être engagée sans approbation formelle du Département ». Les partisans des Français étaient armés pour pallier la faiblesse des troupes d'infanterie de marine, mais les moqueries de l'instituteur sur l'entraînement militaire de 20 autochtones (ils étaient 80 , un an plus tard), laissent peu de doute sur l'usage offensif que la France prévoyait d'en faire : « Pieds nus, avec leurs costumes bigarrés, sur un ordre du chef, ils se mettent à courir tandis que le sergent récite une litanie ininterrompue qui les excite $»$.

Jusqu'en 1895, aucune stratégie alternative ne fut envisagée. Les instructions revenaient, immuables, préconisant « l'apaisement » :

« En laissant le temps faire son œuvre, sans rien brusquer, en étendant peu à peu le cercle de nos partisans, nous verrons sans doute, comme vous le faites justement observer, la résistance s'user et l'ordre se rétablir sans effusion de sang ${ }^{29}$.

Le ministère de la Marine était le premier à blâmer les velléités d'un gouverneur, lorsqu'il souhaitait une action militaire ${ }^{30}$.

En face, la stratégie demeurait la même, de sorte que le face-à-face s'éternisait : « Teraupoo et les chefs ont renouvelé dans les apora les résolutions de ne pas attaquer les Français et de se tenir sur la défensive $»^{31}$.

29 SHD, BB4 1602.

30 ld.

31 Rapport de janvier 1892. 


\section{L'échec de la mission Chessé en 1895}

Chautemps, nouveau ministre des Colonies, décida d'envoyer Chessé, qui avait réalisé l'annexion de Tahiti, pour installer effectivement la souveraineté française à Raiatea. Chessé arriva à Papeete en janvier 1895, avec le titre de Commissaire général de la République ${ }^{32}$. Bien reçu à Huahine et Bora-Bora, il ne parvint même pas à rencontrer les rebelles à Raiatea.

Chessé préférait manier la carotte plutôt que le bâton. Le commandant du Papeete reçut instruction de se montrer particulièrement conciliant avec les habitants de Raiatea qui auraient besoin des services de son bâtiment, y compris les rebelles. Le marin devait se montrer attentif à l'opinion, que l'administration appelait encore "l'état des esprits», comme sous la Restauration, et renseigner Chessé « sur les résultats obtenus par les différents émissaires qu'il y a[vait] envoyés ${ }^{33}$. Un notable tahitien, Édouard Poroï, avait reçu mission de s'introduire à Avera, pour prendre contact avec les rebelles. Mais le commandant du Papeete laissa peu d'espoir d'une évolution favorable. Poroï avait d'abord été interdit d'accès et même frappé d'une amende par les Teraupistes, puis reçu à Avera, mais sans résultat notable : « La situation est toujours la même. Admission ou non admission de nos émissaires ne la modifie pas $»^{34}$.

32 Pierre-Yves Toullelan, Tahiti colonial (1860-1914), Paris, Publications de la Sorbonne, 1987, p. 78.

33 SHD, BB4 1585, le chef de la station au commandant du Papeete, le 17 août 1895.

34 Ibid., rapports du 23 août et 5 septembre 1895 .
Dès le début du mois de septembre, Chessé ne se fit plus d'illusion. Dépité, il usait, dans sa correspondance avec le résident aux ISLV, d'images infantilisantes : "Comme des enfants que l'on isole pour leur permettre de réfléchir au mal qu'ils ont fait, on les isolera du reste du monde ». Il s'essayait à parler leur langue et à entrer dans leur logique : « La malédiction de Dieu sera sur eux, parce que la cause des Français est juste [...] $»^{35}$.

Le maintien de l'état de siège marquait l'échec des négociations. Toutefois, le blocus était désormais restreint aux seuls Teraupistes : le tiers parti acceptait, non pas l'annexion, mais le protectorat. Ce fut suffisant pour que Chessé présente sa mission comme un succès ${ }^{36}$. Mais personne ne s'y trompait, surtout pas l'évêché de Papeete, qui avait proposé son entremise, et collaboré autant que possible, mais considérait que Chessé s'était fourvoyé dans le choix de ses émissaires à Raiatea, notamment lorsqu'ils étaient protestants ${ }^{37}$. Quant au gouverneur Papinaud, il écrivit sans ménagement à son ministre, le 15 février 1896 : « Les choses sont plus difficiles et plus compliquées dans l'archipel des ISLV depuis la mission Chessé ${ }^{38}$.

35 SHD, BB4 1602, lettre de Chessé à Desaille, le résident de France aux ISLV, le $1^{\mathrm{er}}$ septembre 1895.

36 Voir sa lettre au vice-résident du 23 décembre 1895, dans BB4 1603.

37 Archives de l'évêché, lettre au siège de la mission, le 11 novembre 1895.

38 Cité par Jean-François Baré, Tahiti, les temps et les pouvoirs, op. cit., p. 347 (cf. note 9). 
Un an plus tard, alors que la France allait donner l'assaut, Paul Gauguin se moqua abondamment de la tentative de conciliation :

« Le canard Chessé déployant ses ailes a envoyé messager sur messager, fait cadeau aux femmes indigènes de ballons rouges, petites boîtes à musique et autres joujoux (textuel - Je n'invente rien) récité un tas de bêtises de la Bible. Rien ne les a tentés malgré tous les mensonges. Chessé s'est retiré, complètement battu par la diplomatie sauvage $»^{39}$.

De fait, la mission avait essentiellement reposé sur une distribution de cadeaux, dont les passagers de l'Armand Behic avaient aussi profité, au témoignage d'un compagnon de croisière : « Chessé emporte toute une cargaison d'objets variés qui lui serviront à faire des cadeaux là-bas et qu'on juge capables de lui aplanir les voies. Jusqu'à des jouets dont nos enfants profitent les premiers ${ }^{40}$. L'échec de la mission Chessé avait nettement modifié l'état d'esprit des différents acteurs français. Dans leurs rapports, les marins pressaient désormais de recourir à la force et dénonçaient " l'illusion de ceux qui croient encore qu'on peut ramener à nous, autrement que par la force, d'aussi stupides

39 Gilles Artur, « Notice historique du Musée Gauguin de Tahiti suivie de quelques lettre inédites de Paul Gauguin ", Journal de la société des océanistes, vol. 38, $n^{\circ} 74-75$, 1982, p. 4. Lettre de Paul Gauguin à Charles Morice, janvier 1897 (publiée partiellement par la Revue Blanche du $1^{\mathrm{er}}$ novembre 1897).

40 Journal de Charles Rémond, le 18 juin 1895, en ligne sur http://www.messageries-maritimes.org/, site consulté en mai 2013. entêtés $»^{41}$. Les marins déploraient que le tiers parti jouisse d'une impunité qui permettait à ses membres de cultiver des terres sises dans le camp des rebelles et d'en commercialiser le produit à Uturoa, sans se donner la peine de convertir les Teraupistes. "On ne voit se dessiner aucune des améliorations que devait apporter l'établissement du protectorat avec les ralliements nombreux qu'il devait opérer ». En septembre, le commandant du Papeete évoqua pour la première fois, comme une issue probable, le recours à la force " pour reprendre possession complète de notre autorité dans ces îles $»^{42}$.

À Paris, le ministre des Colonies, Lebon, informa son homologue de la Marine qu'ayant « épuisé toutes les possibilités de conciliation », il souhaitait disposer du Duguay-Trouin ${ }^{43}$. Les Affaires étrangères ne semblaient pas approuver le principe de l'intervention, mais ne s'y opposèrent pas. La Marine travailla sur la base d'un rapport de juillet 1896 du commandant de la division navale du Pacifique : il avait été envisagé qu'un corps d'intervention puisse débarquer à Uturoa, en plus de l'infanterie de marine, pour intervenir à Tevaitoa et Avera. En octobre 1896 le Conseil des ministres prit la décision d'intervenir, le Duguay-Trouin fut mis à la disposition. En novembre 1896, l'Aube était de retour de Nouméa où il embarqua une compagnie d'infan-

41 SHD, BB4 1585, IDLS, f. 310.

42 Ibid., rapport du 12 septembre d'E. Deman.

43 Jean-François Baré, Tahiti, les temps et les pouvoirs, op. cit., p. 347 (cf. note 9). 
terie de marine forte de 150 hommes « pour terminer les affaires de Raiatea $»^{44}$. Le commandant du Papeete fut alerté : "Si une dernière tentative de pacification que doit faire le Gouverneur échoue, les opérations contre les rebelles commenceront aussitôt que le DugayTrouin attendu à Tahiti vers le 6 décembre sera prêt à se rendre à Raiatea ».

\section{L'écrasement de la révolte}

Le Papeete, l'Aube et le Duguay-Trouin transportant en plus des équipages, 200 hommes d'infanterie de Marine, 205 artilleurs, des volontaires tahitiens (qui ne furent pas mis en ligne lors de l'assaut) et 150 auxiliaires non armés, soit un millier d'hommes en tout, convergèrent vers les ISLV où les marins avaient balisé le chenal menant de la passe Teava Roa à Avera ${ }^{45}$.

À lire la correspondance de l'administrateur des ISLV, la « rumeur publique » annonçait l'imminence de l'intervention armée. C'est pourquoi il recommanda aux marins de reprendre les mousquetons confiés au tiers parti, « aussi opposés à l'acceptation du pavillon français et à l'autorité française gouvernementale que les vrais rebelles conduits par Teraupoo $»^{46}$. Les marins partageaient ce sentiment :

44 Instructions du 20 novembre 1896 au commandant du Papeete.

45 SHD, BB4 1585, IDLS, rapport du commandant du Papeete, le 24 novembre 1896.

46 Ibid., le 26 novembre 1896.
« Nos protégés d'Avera, bien qu'ils aient été depuis le commencement de l'état de siège jusqu'à ce jour les plus favorisés sous tous les rapports, semblent plutôt vouloir se rapprocher de Teraupoo et du pavillon anglais que de vouloir se rallier aux couleurs françaises $»^{47}$.

Le 27 décembre, un ultimatum de quatre jours fut lancé : les soumis devaient se réfugier sur les quatre motu (îlots) désignés, tandis que les passes étaient barrées par des bâtiments. Ce délai fut employé à une démonstration de force : les troupes furent passées en revue, le 29 décembre, sur la place du temple, à Uturoa, avant de défiler en colonnes.

Les hostilités commencèrent le $1^{\mathrm{er}}$ janvier 1897, selon le plan prévu par les Français : deux colonnes, une par bateau, battirent les deux versants de l'île et convergèrent pour se retrouver sur les crêtes, tout en balayant les vallées, par coups de sondes (cf. carte). Les rebelles, qui refusèrent le combat, furent ainsi refoulés vers le sud. Chaque colonne conserva l'appui de son bâtiment qui arrosa le rivage de son artillerie, pour empêcher les rebelles de remonter vers le nord $^{48}$.

Les deux navires ont commencé par mouiller à Tahaa, pour ouvrir le feu sur les habitations et les embarcations rebelles. Les torpilleurs débarqués «abattent le mât de pavillon au haut duquel était arboré le pavillon anglais,

47 Ibid., le 5 décembre 1896.

48 SHD, BB4 1603, « dossier de Raiatea-Tahaa, expédition de janvier $1897 »$. 


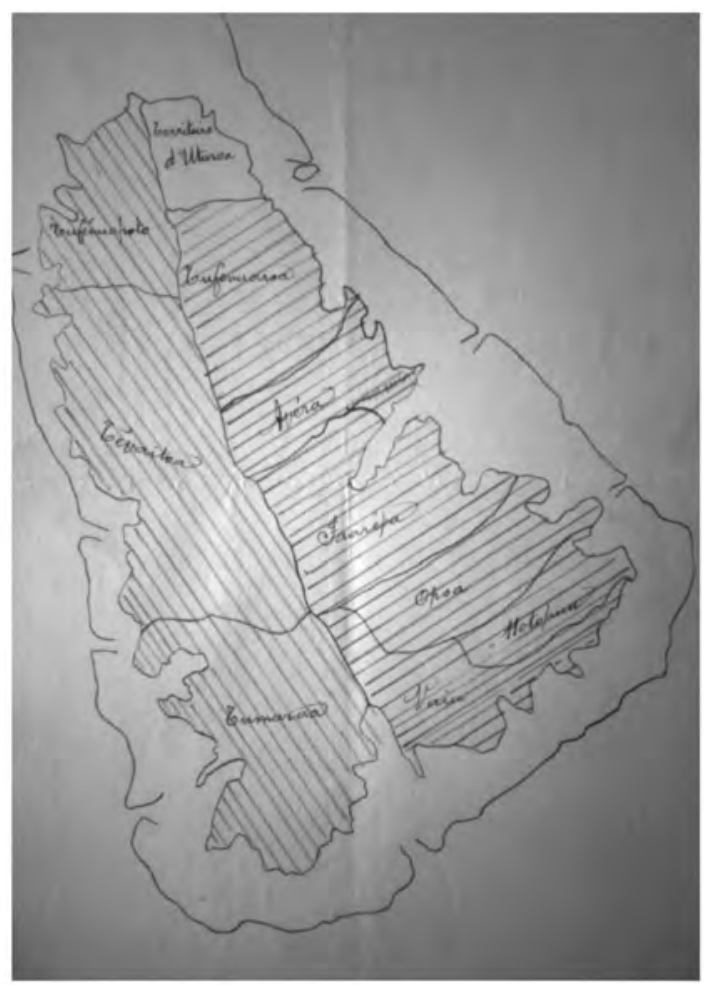

Figure 1. Carte de Raitea pour préparer l'offensive de janvier 1897: les couleurs bleue (rayures horizontales) et rouge (rayures verticales) correspondent aux territoires battus par les deux colonnes de l'infanterie de marine. Seul le territoire d'Uturoa est donc considéré comme pacifié, à cette date (Archives du Service historique de la Défense, BB4 1603).

détruisent le Farehau (maison commune) et 3 cotres, et incendient les habitations des rebelles ». À 14 heures, les navires étaient revenus au soutien de leurs colonnes, à Raitea ; le Duguay-Trouin ouvrit le feu sur les habitations des rebelles et débarqua l'infanterie pour abattre le pavillon anglais. Le lendemain, une première escarmouche opposa les adversaires, lorsqu'une partie de la colonne de l'Aube alla
« occuper le poste avancé des Teraupistes à la pointe Tereva et fouiller les vallées dans le nord de Tevaitoa ». « Tandis qu'elle campait [...], la colonne a été attaquée par une petite reconnaissance teraupiste sans que le feu ait produit de résultats d'un bord ou de l'autre ${ }^{49}$.

49 ld. 
Au matin du 3 janvier, après que des signaux eurent été échangés entre les divers détachements, le village de Tevaitoa fut attaqué sous le feu de l'Aube. À l'entrée du village, les colonnes avancèrent à la baïonnette pour fouiller la pointe de Tevaitoa, où se trouvait un marae, lieu possible d'une embuscade. De fait, le combat principal s'y déroula :

« Les Teraupistes, au nombre d'une quarantaine, blottis dans des retranchements d'un mètre de large et de 1,50 $\mathrm{m}$ de profondeur laissent passer le détachement de l'Aube puis ouvrent le feu en apercevant le gros de la colonne ».

Le retranchement est envahi « et les défenseurs sont tués à la baïonnette tant par les marins que par les soldats », qui coupent la retraite vers la brousse. L'offensive ne coûta aucune perte aux Français, mais causa la mort de 17 rebelles. Le reste des opérations consista en des battues, qui ne trouvèrent plus de résistance mais ne permirent pas de mettre la main sur Teraupoo et sa femme. Il fallut plusieurs semaines pour que des autochtones repèrent des signes de vie, venus d'une grotte, et que l'on se saisisse du couple - le 15 février 1897.

\section{Châtiment et nouvel ordre}

Embarqués sur l'Aube le 28 janvier 1897, 116 hommes, 29 femmes et 24 enfants furent exilés à destination d'Eiao, aux Marquises, et finalement débarqués sur l'île d'Ua Uka, dans la vallée de Katohau. La plupart des exilés venaient de Raiatea, mais une forte minorité était ori- ginaire de Tahaa ; quelques-uns étaient issus de Huahine, et même de Tahiti, sans que l'on sache si la rébellion avait pris une dimension " transnationale », ces résidents d'autres îles pouvant aussi bien être apparentés aux rebelles. Les détenus furent isolés de l'étroite population de l'île marquisienne, dans une vallée où ils s'occupèrent de monter le poste de gendarmerie, de construire leur village et d'étendre les cultures. Leurs premières lettres, où ils confièrent leur mal du pays, dissuadaient leurs proches de les rejoindre dans leur exil ${ }^{50}$.

Le gouverneur choisit un châtiment plus radical, vers le bagne de Nouméa, pour « quelques chefs ou meneurs particulièrement dangereux ». Il aurait aimé châtier les étrangers impliqués dans la rébellion, mais il ne le put, faute de preuves, quoique les marins et l'administrateur aient été convaincus de l'implication de deux d'entre eux, au moins. En plus de l'exil, les rebelles durent abandonner une partie de leurs enfants. L'administrateur des ISLV s'inquiéta des adoptions forcées et refusa qu'un jeune homme de dix-neuf ans emmène, pour le compte de sa famille, un " petit enfant d'une famille rebelle », alors que Tati Salmon, une personnalité tahitienne, avait déjà reçu « cinq petits rebelles qui lui [avaient] été confiés sur sa demande». Il relayait les rumeurs qui couraient à Uturoa : «M. Tati Salmon aurait payé le gouvernement français pour se faire livrer ces enfants comme esclaves ».

50 Ibid., lettres de février 1897. 
La nomination de nouveaux chefs de district, le 23 janvier, ayant « autorité absolue sur tous les indigènes de leur district quels qu'aient été antérieurement les dignités, grades, emplois ou fonctions de ces dits indigènes », marqua le nouvel ordre français. Sur proposition du chef de la division navale du Pacifique, l'ancienne reine du protectorat d'Avera reçut une pension annuelle de 2400 francs, au titre de son (tardif) ralliement. Mais la France refusa sa demande de " pensions pour divers membres de sa famille », et le gouverneur ajouta :

« Je vous laisse d'ailleurs le soin, si vous le jugez convenable, de prévenir Tuarii que si elle nous crée la moindre difficulté sa pension lui est retirée, car elle ne la doit qu'à notre extrême bienveillance en sa faveur $»^{51}$.

\section{Teraupoo, le rebelle de Raiatea}

S'il est difficile d'estimer l'autorité de Teraupoo au-delà de ses partisans, qui représentaient environ le tiers des habitants, les papiers saisis par les marins français à l'issue de l'assaut donnent à penser que le refus de la souveraineté française débordait largement au-delà de sa figure.

Une lettre demandant la protection de la GrandeBretagne le montre, puisque l'administrateur des ISLV qui a émargé à l'intention du chef de la division du Pacifique, la liste des noms pour indiquer leur parti, a indiqué pour la moitié

51 Ibid., 15 février 1897. d'entre eux qu'ils étaient théoriquement des protégés de la France ${ }^{52}$.

Il est difficile de se représenter la place que Teraupoo occupa dans l'imaginaire politique des habitants de Raiatea, lors de son exil, puis à son retour. Quel crédit accorder au récit romancé de Jean Dorsenne, fondé sur des sources orales, lorsqu'il imagine son retour pitoyable d'exil ?

"Teraupoo revint à Raiatea en 1906. Il ne subsis-
tait aucun vestige des troubles passés. L'autorité
française s'étendait dans le pays, et nul ne sem-
blait se souvenir des événements malheureux qui
avaient éclaté huit ans auparavant. [...] Il vécut
dans l'isolement, fréquentant peu ses voisins,
parlant le moins possible ${ }^{53}$ ? Il y avait certainement un écart important entre le trentenaire, qui avait tenu tête pendant dix ans à la souveraineté française, et l'homme déjà âgé qui revint d'exil ; c'est aussi ce que nous dit une légende qui nous instruit sur la postérité de sa figure : « De nos jours, les habitants de Ra'iateaa continuent à propager la rumeur que Teraupoo aurait été tué par les Français et que la personne rentrée d'exil ne serait pas le chef insurgé ${ }^{54}$.

De fait, sa légende a continué de produire des effets historiques, lorsque les mouvements

52 Ibid., 14 février 1897. Probablement datée de 1896, soit après le nouveau pacte de protectorat passé avec la reine et le tiers parti lors de la mission Chessé.

53 Jean Dorsenne, Un fils de cannibales, op. cit. (cf. note 16).

54 Anne-Lise Pasturel, « Raiatea 1818-1945 », op. cit., p. 358, note 306 (cf. note 8). 
indépendantistes tahitiens les plus radicaux, dans les années 1970, se sont réclamés du « martyr » pour mener leurs actions violentes (assassinat d'un métropolitain). Les échos les plus récents de la guerre de ISLV se font entendre sur un ton plus pacifié, à travers une forme de « patrimonalisation » du souvenir. Une troupe de danse a, par exemple, choisi de raconter la guerre des ISLV, en 2009, lors du Heiva (festival annuel, comprenant notamment des spectacles de danses traditionnelles). Enfin, par un étonnant résumé de la dimension politique du renouveau identitaire polynésien, le nom du rebelle est revenu sous la forme d'un prénom, porté notamment par la légende vivante de la pirogue à voile tahitienne, Teraupoo Richmond. 